\title{
Mejoramiento del proceso de suavizado de la fibra de cabuya para elaborar géneros textiles
}

\section{Improvement of the softening process of the cabuya fiber to elaborate textile goods}

\author{
Diego Gustavo Betancourt Chávez \\ Dolores Krupskaya Salazar Garcés \\ Universidad Técnica de Ambato, Ecuador
}

Autor para correspondencia: diegogusbetancourt@hotmail.com; dbetancourt@uta.edu.ec Fecha de recepción: 05 de Julio de 2017 - Fecha de aceptación: 12 de Agosto de 2017

Resumen: El proyecto de Mejoramiento del proceso de suavizado de la fibra de cabuya tiene como propósito impulsar la utilización de la fibra de la cabuya como materia prima para la elaboración de géneros textiles que puedan ser utilizados en la confección de prendas de vestir. Los procesos de suavizado de la cabuya pese a ser muy sencillos no han llegado a ser óptimos para obtener fibras apropiadas para la elaboración de géneros textiles. El proceso más utilizado, y con el que se han obtenido los mejores resultados se realiza después de la tintura, para lo cual se emplean suavizantes naturales como la sábila ó químicos como el soflan o rinse. En la parte experimental se busca determinar la relación óptima entre la cantidad de suavizante y la suavidad de la fibra, así como también se trabaja con el uso de productos que no han sido desarrollados como suavizantes pero pueden ayudar a esta función como lo son las enzimas ácidas, para obtener una fibra que pueda ser utilizada en la elaboración de géneros textiles que puedan ser aplicados en indumentaria. Se trabajará con fibras procesadas en diferentes plantas productoras, a las cuales se les aplica el tratamiento experimental con diferentes procesos de suavizado: micro y macro emulsión de silicona, ácido graso, y aplicación de encimas tanto ácidas como neutras. Una vez concluida la prueba, se analizarán ventajas, desventajas y sobre todo, verificar cuál de los procesos da como resultado un mejor tacto y por ende brinda la mejor opción para la elaboración de géneros textiles. Con esta investigación se espera mejorar el proceso de suavizado de la fibra de la cabuya, lo que reportaría beneficios tales como versatilidad de uso, mayores oportunidades de trabajo para las personas que laboran con ella y menor impacto ambiental en la producción textiles

Palabras Clave: género textil; cabuya; enzimas; suavizante

Abstract: The Improvement of the softening process of cabuya fiber is aimed to promote the utilization of cabuya fiber as raw material for the fabrication of textile products that can be used in the manufacture of garment. Although very simple, cabuya softening processes have not yet become an optimal for obtaining suitable fibers for the elaboration of textile products. The most used process, and with which the best results have been obtained, is done after dyeing in which natural softeners such as aloe vera and chemical softeners such as soflan or rinse are utilized. Nevertheless, in the experimental section, it is sought to determine the optimal relationship between the amount of softener and the softness of the fiber, as well as the use of products that have not been developed as softeners but can work as the enzymes in order to acquire a fiber which can be used in the manufacture of textile fabrics, applied in clothing. On face of it, it will be 
necessary to apply an experimental treatment to production-plants-processed fibers by different ways: micro and macro emulsion of silicone, fatty acid, and application of both acidic and neutral enzymes. Once the test is finalized, advantages and disadvantages will be analyzed, however to determine which one of the processes applied works best in terms of tact and, therefore, offers the options for the elaboration of textile products. To sum up, this research is expected to improve the smoothing process of cabuya fiber, which would bring benefits such as versatility of use, greater job opportunities for people working on it and less environmental impact on textile production Key words: textile product; cabuya; enzymes; softener

\section{Introducción}

El Centro de Información e Inteligencia Comercial argumenta que la fibra de la cabuya es una materia prima poco utilizada en la industria de la moda para la confección de prendas de vestir, debido a que es muy difícil de trabajar por su dureza y aspereza característica; quedando su empleo casi exclusivamente para la elaboración de sogas sacos y artesanías.

Desde hace cientos de años se han aprovechado las hojas de la cabuya para obtener la fibra textil debido a su resistencia, durabilidad y consistencia áspera. (Cardona Franco 2006). La cabuya está calificada como una fibra vegetal áspera y dura dentro de la clasificación de las fibras naturales (Jurado y Sarzosa SF). Los procesos de suavizado de la cabuya son muy sencillos y no han llegado a ser óptimos para obtener fibras de cabuya apropiadas para la obtención de géneros textiles.

El proceso de suavizado de la cabuya más utilizado y con el que se han obtenido los mejores resultados -en lo que a tacto se refiere- se lo realiza después de la tintura, para lo cual se utilizan suavizantes naturales como la sábila o químicos como el soflan o rinse, con estos productos se deja a la fibra sumergida durante 10 minutos y luego de esto se pone a secar la fibra (Orejuela 1996).

Se han realizado hilados de calibre muy fino de fibra de cabuya, pero aún no se han desarrollado procesos de suavizado, con el cual esta fibra se vuelva confortable al contacto con la piel.

\section{Revisión Literaria.}

La fibra de la cabuya proviene de las hojas de la planta con el mismo nombre, conocida también con el nombre de agave o fique es una planta sumamente rústica, que se viene explotando en Ecuador desde la época pre Incacica. En el campo es una planta que tiene muchas funciones, por ejemplo se la utiliza para elaboración de géneros textiles y cuerdas, como detergente, combustible, división de tierras, como alimento del ganado y de las personas, de sus flores se obtiene las alcaparras. (Bastidas \& Orozco, 2013)

En el Ecuador esta planta se cultiva principalmente en la sierra centro, en las Provincias de Imbabura, Pichincha, Cotopaxi, Tungurahua y Bolívar, debido a la eficiencia en sus raíces y la característica de desarrollarse en laderas con pocos nutrientes se convierte en un sistema económico e ideal para la conservación del suelo. (Yepez \& Esparta, 2009) 
En la industria textil y de la moda sus aplicaciones son limitadas al área de cordelería debido a que por su estructura la cabuya es una fibra poco confortable y áspera al contacto con la piel, pero debido a su gran resistencia es utilizada en alpargatas, shigras, tapetes, alfombras y principalmente en artesanías (Ramirez, 2014).

En el Ecuador se distingue la variedad de cabuya blanca y negra las mismas que dependiendo de su estructura se pueden clasificar en:

Macrofilia: Es una planta de tallo corta cuya altura aproximada es de $30 \mathrm{~cm}$, sus hojas presenta un color verde de apariencia cóncava, con la característica que en el haz de las mismas tiene una textura lisa, al contrario en la parte posterior de la hojas se presenta una estructura áspera, el ancho de la hoja oscila entre los 8 y $14 \mathrm{~cm}$ y la longitud de las mismas va desde los $1.5 \mathrm{~m}$ hasta $\operatorname{los} 2 \mathrm{~m}$, condición que permite que sus hojas sean aptas para la obtención de fibra, al hablar de las espinas ubicadas a lo largo de la hoja son de color rojo con apariencia curva su tamaño es pequeño y la distancia que existe entre las espinas puede variar entre los 40 y $80 \mathrm{~cm}$, en lo que a producción de fibra se refiere del peso total de la hoja se obtiene solo del 3 al $4 \%$ (Yepez \& Esparta, 2009).

Andina: Este tipo de planta presenta un tallo muy corto por lo que su altura puede llegar máximo hasta los $20 \mathrm{~cm}$, la mayoría de sus hojas son de color verde con apariencia un poco cóncava casi planas, su textura en los dos lados de la hoja es lisa, en lo que al ancho se refiere las hojas de esta variedad de cabuya pueden llegar a tener entre 10 y $15 \mathrm{~cm}$, y su longitud puede variar entre los $1.2 \mathrm{~m}$ hasta $1.7 \mathrm{~m}$, razón por la cual estas hojas son aptas para la obtención de fibra, en el caso de las espinas se encuentran alojadas en el borde externo de las hojas y la dirección de la punta de las mismas va hacia la punta de la hoja, el tamaño de las espinas varian entre los 5 y $8 \mathrm{~mm}$ y la distancia ente espina y espina varía entre los 15 y $20 \mathrm{~cm}$, en lo que a producción de fibra se refiere es un poco bajo ya que al año de una planta máximo se obtiene un kg de la misma (Bastidas \& Orozco, 2013).

Humboltiana: En este tipo de cabuya se aprecia un tallo muy largo que varía entre 1 y 3 metros de longitud y en su punta se ubican los frutos de los cuales se pueden obtener las alcaparras, al contrario de las dos especies anteriores esta presenta un color verde agrisado en sus hojas su estructura es casi plana y pueden llegar a medir de 12 a $15 \mathrm{~cm}$ de ancho, mientras que de largo su tamaño varía entre 1 a 1,7 m de longitud, debido a esta característica se convierten en idóneas para la producción de fibra de cabuya tomando en cuenta que la longitud recomendable para que la fibra sirva como materia prima textil es de $1.3 \mathrm{~m}$ como mínimo; en lo que se refiere a las espinas son marginales es decir se ubican en el borde externo de la hoja a una distancia entre los 25 y $65 \mathrm{~cm}$ entra espinas y su tamaño varia de 2 a $5 \mathrm{~mm}$, esta clase de cabuya blanca tiene una producción de $600 \mathrm{~kg}$ a 1 tonelada anual por cada hectárea de cultivo convirtiéndose en la de mayor producción de las existentes en el Ecuador (Yepez \& Esparta, 2009).

Al hablar de la cabuya negra perteneciente al género del agave se menciona que posee gran cantidad de pulpa y jugo razon por la cual no es recomendable para la producción de fibra textil ya que su extracción es complicada y el rendimento no es óptimo, es por ello que los indigenas de la sierra ecuatoriana utilizan este tipo de cabuya para alimentacion de su ganado, 
como detergente y para extraer el chaguarmisque que es un nectar obtenido del corazón de la planta el mismo que al ser fermentado es consumido como licor (Jurado \& Sarzosa, 2009).

\section{Cultivo}

Para su cultivo los suelos pueden variar entre arcilloso y arenoso debido a que es una planta que se adapta a condiciones variadas, pero para obtener resultados de mayor impacto es recomendable cultivarla en suelos arenosos ricos en calcio, adicionalmente para aumentar su desarrollo es necesaria la presencia de Magnesio, Fósforo y Potasio, con relación al clima los factores más importantes son Luz, humedad atmosférica, temperatura y agua, en el Ecuador las condiciones óptimas son:

$\checkmark$ Temperatura entre los 18 y $24^{\circ} \mathrm{C}$, los mismos que se consiguen a una altura entre los 1000 y 2000 metros sobre el nivel del mar. (Aguilar, Ramírez, \& Malnagon, 2007)

$\checkmark$ Humedad relativa debe encontrarse entre el 50 y el $70 \%$ en caso contrario la planta está expuesta a un ataque patológico (Jurado \& Sarzosa, 2009).

$\checkmark$ Precipitación: las precipitaciones pluviales pueden ir de 1000 a $1600 \mathrm{~mm}$ anuales (Ministerio de Agricultura y Ganaderia, 2008).

$\checkmark$ Luminosidad: de acuerdo a estudios realizados se determina que la cantidad de luminosidad diaria debe ser como mínimo de 5 a 6 horas. (Aguilar, Ramírez, \& Malnagon, 2007)

$\checkmark$ La acidez: El Ph óptimo para el desarrollo de la cabuya es de ligeramente ácido de 5 a 7 con lo que se favorece el crecimiento radicular de la raíz de la planta (Aguilar, Ramírez, \& Malnagon, 2007).

\section{Cosecha}

La cosecha se empieza con el proceso conocido en el campo como desbajerada o pre cosecha, la misma que se realiza con unos meses de anterioridad para este procedimiento se recomienda cortar las hojas que se encuentran en contacto con el suelo ya que estas tienen un 90\% de probabilidad de que se dañen (Sanchez, 1990).

El corte consiste en cortar periódicamente de la planta cierto número de hojas con un cuchillo o machete, dependiendo de la altura a la que se encuentre el cultivo de cabuya el primer corte se lo realiza entre los 2 años hasta los 4 años y medio, asi por ejemplo para una altura de 1700 metros sobre el nivel del mar el corte se lo puede realizar entre los 2 años y los 3 años, además a esa altura una planta promedio puede producir más o menos 50 hojas por corte, en su defecto una planta de cabuya que se encuentre cultivada a los 2200 metros sobre el nivel del mar el primer corte se los realiza entre los 3,5 años y los 4,5 años obteniendo un promedio de cosecha de 20 hojas por planta, para realizar el corte se debe tomar en cuenta los siguientes aspectos:

1. Se debe cortar solamente las hojas maduras.

2. Se pueden efectuar hasta un máximo de tres cortes en el año.

3. Es recomendable realizar el corte lo más cerca posible al tallo. 
4. Para asegurar una buena calidad de fibra textil es recomendable recortar las hojas de 15 a $20 \mathrm{~cm}$ de la base para aumentar la calidad de la fibra y disminuir el desperdicio

5. Realizar el corte sin herir a las hojas que aún quedan en la planta y finalmente despuntar y despalmar las hojas antes de empezar el desfibrado (Sanchez, 1990).

\section{Desfibrado Manual}

Antes de empezar con el desfibrado se debe tomar en cuenta que una vez que las hojas son cortadas tienen que pasar al proceso de desfibrado antes de las 36 horas siempre y cuando se encuentren en un lugar en el cual no exista lluvia ni sol, en caso contrario las células de las hojas de la cabuya se mueren y empiezan a secarse complicando el proceso del desfibrado (Yepez \& Esparta, 2009).

El proceso de desfibrado empieza por el macerado, continua con el golpeado y finalmente con el raspado hasta dejar libre la fibra de la hoja, estos pasos se los cumple tanto en el proceso artesanal como en el proceso industrial. (Yépez \& Esparta, 2009)

Macerado: consiste en realizar atados de 5 a 10 hojas de cabuya y colocarlas dentro de un recipiente cerrado que contenga agua por un tiempo aproximado de 1 mes. (Jurado \& Sarzosa, 2009)

Golpeado: consiste en dar golpes a los atados de hojas de cabuya hasta eliminar por completo la pulpa y la goma que existe en ella (Jurado \& Sarzosa, 2009).

Desfibrado: Finalmente se peina a las fibras que quedan para obtener el material textil adecuado (Yépez \& Esparta, 2009).

\section{Desfibrado industrial}

Este es el método de desfibrado más utilizado en la actualidad con el empleo de una máquina que funciona con un motor, en el interior de la máquina existe un tambor de $40 \mathrm{~cm}$ de diámetro y en su superficie consta de 15 a 20 cuchillas las mismas que ayudan a raspar, golpear y limpiar las hojas de la cabuya, limpiando eficientemente la goma y la pulpa propias de las mismas, este procedimiento se comienza realizando una clasificación de las hojas tomando en cuenta el grosor de las mismas, con el fin de calibrar la máquina de acuerdo al espesor del atado de hojas, para este desfibrado se empieza desde la mitad de la punta de la hoja luego de lo cual se continua dando la vuelta a la hoja y se introduce la parte de la base de la misma. (Ministerio de Agricultura y Ganadería, 2008).

\section{Características físicas de la fibra de cabuya}

La fibra de cabuya es considerada como una fibra dura, debido a que sus hojas tienen en su estructura nervadura de fibras principales o fibrillas que se fusionan entre sí por la goma propia de la planta y esta a su vez proporciona la rigidez y aspereza de la misma, a esta goma se la conoce como el cemento vegetal, las fibrillas tienen una longitud de 2 a $6 \mathrm{~mm}$ por lo que son 
muy cortas y la unión de estas forman largos filamentos los mismos que son conocidos con el nombre de fibras o hebras de cabuya (Hollen, 2004)

Estas fibras o hebras cumplen con la función de dar rigidez y resistencia a las hojas, también sirven de base para los conductores de sabia para toda la hoja por todas estas funciones mecánicas a estas fibras se las conoce como fibras estructurales, cuando se realiza un corte transversal de la hoja se observa claramente que estos ejes de fibras se encuentran en mayor cantidad en los bordes y en la base de las hojas dejando muy poca cantidad de los mismos en la punta y en el centro de la hoja, la cantidad de fibras por cada hoja de la cabuya así como su distribución depende de la especie, por lo que pueden distinguirse claramente tres clases las mismas que son:

Fibras mecánicas.- Existen en mayor cantidad en los bordes externos de la hoja, también pueden distribuirse por el parénquima foliar; casi nuca cumplen la función de convertirse en tejido conductor es decir el medio de transporte de la sabia; tiene la característica que en su sección transversal se observa la forma de una herradura y su longitud es muy variable la misma va desde milímetros hasta metros, estas fibras son de un calibre fino a tal punto que se parten o dividen longitudinalmente en el proceso de desfibrado (Morales, 2002).

Fibras sueltas.- Estas son las fibras de mayor número en la zona central de la hoja de la cabuya en su sección transversal se observa la forma de la luna creciente, al ser de las fibras más largas de la hoja y de buena resistencia son muy útiles para convertirse en fibras con aplicaciones textiles y por ello son consideradas de gran valor comercial (Hollen, 2004).

Fibras del xilema.- Se encuentran en la línea media de la hoja, y su sección transversal presenta forma de luna creciente irregular; su longitud es variable. Las paredes de las células que componen estas fibras son muy finas y frágiles, por lo cual se fragmentan durante el proceso de desfibrado y pasan a constituir gran parte de residuo (Hollen, 2004).

Para determinar las características física de la fibra de cabuya se deben analizar varios aspectos y factores como son tipo y calidad de suelo, clima, humedad, región, la especie y procesos mecánicos a los cuales han sido sometidas las hojas de la cabuya, así por ejemplo:

Longitud de fibra.- La longitud promedio está establecida en $130 \mathrm{~cm}$ pero puede variar entre 50 y $300 \mathrm{~cm}$ dependiendo su procedencia

Color.- El crema es el color característico de las fibras naturales vegetales y por ende de las fibras de la cabuya.

Brillo.- Es una fibra que puede considerarse como muy lustrosa.

Tacto.- Al contacto con la piel es áspera y dura.

Producción.- En promedio una planta puede producir $1 \mathrm{~kg}$ de fibra por cada año de cultivo.

Calibre.- (tex) 22.63

Resistencia.- (RKM) 24.41

Elongación.- 0,3 a 0,4 \%

Fuente:(Hollen, 2004) 


\section{Lavado}

Para realizar el proceso de lavado es necesario disponer de recipientes con agua totalmente limpia y adicionar de 5 a $7 \mathrm{~g} / \mathrm{l}$ de detergente con el objetivo de blanquear y limpiar la fibra, aunque este proceso aumenta el costo del producto es necesario para mantener y preservar la calidad del mismo, para desarrollar el proceso de lavado se debe sumergir en los recipientes con agua y detergente por un lapso de 15 horas y seguidamente se debe someter a un lavado con agua corriente a la fibra para eliminar los restos de corteza, pulpa, espinas y detergente que se encuentre en la fibra, este lavado también se lo puede realizar en agua corriente para disminuir el tiempo (Orejuela, 1996).

\section{Secado}

Este proceso se lo puede realizar de dos formas: artificialmente y de una manera natural, a nivel industrial se utiliza el secado artificial el cual se compone de 2 máquinas una centrifuga en la cual se elimina el exceso de agua existente en la fibra y la secadora que funciona mediante aire caliente es decir debe existir circulación de aire a una temperatura de 100 a $200{ }^{\circ} \mathrm{C}$, (Ministerio de Agricultura y Ganaderia, 2008)

\section{Descrude}

\section{Preparación y acabado de la materia prima}

Este proceso se lo realiza con el objetivo de eliminar las impurezas y residuos vegetales de la fibra además de los aceites propios de la misma, adicionalmente en este proceso se puede mejorar el grado de blanqueo de la fibra razón por la cual para su desarrollo es necesario utilizar Hidróxido de Sodio que cumple con la función de interactuar con las macromoléculas de la fibra para conseguir eliminar todos los aceites grasas y ceras presentes en la fibra, los detergentes deben cumplir con tres funciones específicas en la fibra las mismas que son: separación de impurezas de la fibra, dispersión de la impureza en la solución detergente y la estabilización de la impureza dispersada, finalmente el Agua Oxigenada cumple la función de agente decolorante de la fibra y debe cumplir con las funciones de blanqueo de la fibra, adicionalmente existen otros productos que pueden cumplir con la misma función pero son de un carácter mucho más contaminante, se debe tomar en cuenta varios factores que intervienen en el mismo, asi por ejemplo, la concentración de los productos va a variar de las condiciones de la fibra; la temperatura se debe mantener entre 80 y $90^{\circ} \mathrm{C}$ por un lapso de tiempo no menor a los 60 minutos, en un ph alcalino y con una relación de baño mínima de 1:15, durante todo el proceso es recomendable mecer de manera continua (Cegarra, 1979).

\section{Neutralizado}

En este proceso simplemente se quiere conseguir que el $\mathrm{Ph}$ de la fibra después del proceso de descrude se encuentre neutro, para lo cual se utiliza ácido acético hasta conseguir un $\mathrm{Ph}$ de 5 con el objetivo que al final del proceso la fibra tenga un ph de 7 (Cegarra, 1979).

\section{Suavizado}


Se debe mencionar que antes de empezar con el proceso de suavizado de la fibra es necesario determinar si la fibra va a necesitar ser tinturada o no ya que si se va a tinturar la fibra se debe realizar antes del proceso de suavizado (Manfred, 2015).

El proceso de suavizado es un acabado que brinda al género textil de un tacto más suave haciéndolo flexible y liso, para obtener esta característica se pueden utilizar varios productos que se los puede clasificar en naturales y sintéticos (Manfred, 2015).

Suavizantes naturales.- Al ser la cabuya una fibra dura necesita de un suavizado para que pueda ser maleable y así se elaboren géneros textiles con esta materia prima, para este objetivo a nivel natural se utiliza la sábila como suavizante natural, para lo cual se sumerge la fibra en una solución de sábila y agua en una relación de $1: 4$ durante 30 minutos, después de lo cual es necesario sacudir fuertemente la fibra, dejarla secar sin exponerla directamente al sol y procurando que la fibra no se doble (Bastidas \& Orozco, 2013).

Suavizantes Sintéticos.- Dentro de los suavizantes sintéticos podemos encontrar suavizantes catiónicos, aniónicos y anfotéricos. (Manfred, 2015)

Suavizantes Catiónicos.- Este tipo de suavizantes existen desde los años 30 donde se descubre que varios compuestos catiónicos como auxiliares de tintura, detergentes, etc. eran muy sustantivos al contacto con las fibras de celulosa, en un inicio fueron utilizados para mejorar la solidez de los colorantes directos a las pruebas de lavado. (Manfred, 2015)

En la parte química este tipo de suavizantes son compuestos cuaternarios de amonio con cadenas grasas, sales de amido aminas, grasas cuaternarias e lmidazolinas, razón por la cual presentan buena compatibilidad con los reticulantes utilizados en los acabados de las fibras celulósicas, adicionalmente se convierten en excelentes alisantes de fibra. (Manfred, 2015)

Este tipo de suavizantes tienen tanta versatilidad que se empezaron a utilizar en las formulaciones de combinación de detergentes y suavizante para ser utilizados en el último enjuague de las máquinas de lavar ropa, convirtiéndose este en el método de mayor difusión de suavizado a nivel de la industria textil (Morales, 2002).

Para su aplicación es necesario controlar el ph a un valor de 5 , a una temperatura de $50^{\circ} \mathrm{C}$ durante un tiempo de 20 minutos luego de lo cual no es recomendable enjuagar ni exprimir y sobre todo el secado debe realizarce sin que se exponga al sol la fibra para evitar el amarillamiento y el resecamiento de la fibra, cabe indicar que la aplicación de este tipo de suavizantes provoca la modificación en algunos casos de sus propiedades físicas, sobre todo fibras de carácter queratinoso como son las de los pelos animales y de una forma menos agresiva sobre las fibras celulósicas, es así como el volumen o cuerpo de la fibra se incrementa a la acción de este tipo de suavizantes, la resistencia a la abrasión se aumente ya que la lubricación de la fibra disminuye la resistencia a la fricción, en cuanto a la resistencia a la rotura con la aplicación de este tipo de suavizantes, la fibra puede aumentar su resistencia hasta en un $30 \%$. Por el efecto del suavizante, pueden verse modificadas las siguientes, en cuanto a la resistencia al desgarro su porcentaje aumenta ya que por la lubricación de la fibra obtenida por este tipo de suavizante su capacidad de estirarse sube hasta en un 15\% (Manfred, 2015). 
Suavizantes Aniónicos.- Este tipo de suavizantes es recomendado para su aplicación vía foulard es decir por impregnación y no por agotamiento, tienen como característica principal brindar propiedades antiestáticas a los textiles en mayor cantidad a los suavizantes catiónicos, en cuanto a la permeabilidad de las fibras tratadas con este tipo de suavizantes mejora considerablemente y por ende la capacidad de humectación es muy alta con este tipo de suavizantes, una de las desventajas del uso de este tipo de suavizante es que no mejora la suavidad de la fibra al tacto y tampoco ayuda a que la fibra se hinche y produzca el efecto de incrementar el cuerpo de género textil (Morales, 2002).

Para su aplicación es necesario realizarlo en un ph ligeramente alcalino 8 por 2 pasadas en foulard a una temperatura de $40^{\circ} \mathrm{C}$, es importante recalcar que para secar el género textil tratado con este tipo de suavizantes es recomendable realizarlo a $85^{\circ} \mathrm{C}$ en un secador de aire caliente (Manfred, 2015).

Este tipo de suavizante es recomendable utilizarlo en fibras queratinosas y sintéticas, es decir no es recomendable utilizar este tipo de suavizante sobre fibras celulósicas porque en su composición química su base es un éster de amida (Morales, 2002).

Suavizantes no ionicos.- Poseen, en general, estructura parecida a la de los catiónicos, pero para ser solubles en agua se debe realizar una reducción de óxido de etileno, lo que provoca que en la molécula se genere la característica de ser soluble en agua, su aplicación es casi específica para rayón viscosa y se lo puede aplicar via agotamiento o por foulard, como característica es que cuando se aplica este producto a la fibra esta adquiere un ligero carácter anti estático, no interfieren en el proceso de tintura y no afectan en las solideces de los colorantes, su aplicación se la realiza en un ph neutro para mantener las características y su máxima eficacia se produce a los $50^{\circ} \mathrm{C}$ por un tiempo de 30 minutos, no presentan problemas al tacto y no le dan el carácter impermeable al género textil aunque tampoco se consigue la característica de cuerpo sobre el género textil (Manfred, 2015).

Suavizantes Anfotéricos.- Son suavizantes que pueden ser aniónicos, catiónicos o no iónicos, según el $\mathrm{Ph}$ del medio. Se posee escasa experiencia sobre los mismos. Son aniónicos en medio alcalino, catiónicos en medio ácido y no iónicos en medio neutro, por lo cual se supone que tienen una constitución parecida a la de los aminoácidos conteniendo un radical graso. El grupo solubilizante será seguramente el sulfónico y poseerán un grupo amino o imino, que en medio ácido podrá formar la correspondiente sal de amonio cuaternaria (Cegarra, 1979).

Se puede aplicar a todas las fibras, son compatibles con todos los agentes de apresto, no amarillan el blanco ni siquiera sometiéndolo a polimerización, no alteran los matices de los colorantes directos y poseen ligero efecto antiestático. No tienen afinidad para las fibras, pero si se quiere originarla, basta operar en medio ácido. Son aptas para suavizar en aparatos de tintura, distribuyéndose uniformemente por el material y, si se quiere agotar, basta acidificar ligeramente con ácido orgánico (Manfred, 2015).

\section{Metodología}


Para este proyecto que pretende mejorar el proceso de suavizado de la fibra de la cabuya para la elaboración de géneros textiles, se ha optado por un investigación experimental, ya que la misma permitirá determinar la relación entre la suavidad de la fibra y su uso en prendas de vestir.

Se trabajará con fibra de cabuya procesada en las diferentes plantas productoras del país. Una vez seleccionadas muestras representativa de fibras, se les aplicará el tratamiento experimental con diferentes procesos de suavizado: con suavizantes Cationicos, Anionicos y Anfotéricos, a afín de analizar sus ventajas, desventajas y sobre todo verificar con cuál de los procesos se obtiene un mejor tacto de la fibra y determinar si con estos suavizantes se obtiene un tacto confortable al contacto con la piel.

El diseño experimental escogido para este proyecto es el de grupo de control pretestpostest. La ejecución de este diseño implica tres pasos a ser realizados por parte del investigador:

Medición previa del suavizado de la fibra de cabuya.

Aplicación de la elaboración de géneros textiles para la confección de prendas de vestir con la mejor opción de fibras de cabuya suavizadas. Nueva medición del suavizado de la fibra de la cabuya después del proceso mejorado de suavizado.

Este diseño usa un pretest, que de algún modo proporciona información acerca de la muestra con la que se realiza el estudio y de esta manera se estaría controlando la selección como variable. Las variables a considerar serán: el suavizado de la fibra de cabuya y la obtención de géneros textiles para la elaboración de prendas.

La elaboración de los instrumentos para realizar el experimento y medir sus resultados tiene como premisa anteriores procesos de suavizado en los cuales se revisarán parámetros sobre todo de tacto y maleabilidad de la fibra de cabuya.

El proceso empírico que se sigue actualmente es el siguiente: se toma un tamo de cabuya (bulto), y se lo limpia de las impurezas y restos de la penca y las espinas se deja secar la fibra durante dos días al sol, luego en una relación de baño de 1 a 50 se coloca el 15\% de NaOH (Sosa Cáustica) con relación al peso y se la hierve durante 120 min y se deja secar. Explored (1998).

El proceso de suavizado propuesto se realizará de la siguiente manera: se tomará una muestra de fibra y se la colocará en un recipiente con un relación de baño de 1 a 20, luego se le aplicará distintas relaciones de suavizante y se lo someterá a condiciones variables de tiempo, a una temperatura de $50{ }^{\circ} \mathrm{C}$ y en un $\mathrm{Ph}$ 4.5. Después se la dejará secar y se analizará el tacto de la fibra de cabuya suavizada. Se debe mencionar que el género textil se lo realizará únicamente con la mejor muestra obtenida después de los ensayos.

La preparación del procedimiento para recoger los datos del experimento estará en dependencia de las mejoras al proceso de suavizado de la fibra de cabuya. 
Los resultados se presentarán en forma física y estadística, de modo que se pueda apreciar claramente el efecto de suavizado de la fibra de cabuya y la oportunidad para elaborar géneros textiles de uso en la confección de prendas de vestir.

Para el estudio experimental se realizará el siguiente proceso:

De la investigación bibliográfica desarrollada se obtiene las características de la fibra de la cabuya en la que se determina los procentajes dela compsicion química de la misma, asi como la forma de cultivo cosecha y procesamiento de la misma con el fin de obtener una fibra apta para la elaboración de cordelería que es en lo que actualmente se la utiliza.

\section{Composición química de la fibra de Cabuya}

\begin{tabular}{ll}
\hline Compuesto químico & Porcentaje \\
\hline Celulosa & $73.8 \%$ \\
Lignina & $11.3 \%$ \\
Pentosanos & $10.5 \%$ \\
Cenizas & $0.7 \%$ \\
Humedad Ceras y Grasas & $2.7 \%$ \\
\hline
\end{tabular}

Fuente:(Hollen, 2004)

\begin{tabular}{|c|c|c|c|}
\hline \multicolumn{2}{|c|}{ ALGODÓN } & \multicolumn{2}{c|}{ CABUYA } \\
\hline COMPUESTO & $\%$ & COMPUESTO & $\%$ \\
\hline CELULOSA & $96 \%$ & CELULOSA & $73.8 \%$ \\
\hline ACEITES Y GRASAS & $0.6 \%$ & $\begin{array}{c}\text { RESINAS, ACEITES } \\
\text { Y GRASAS }\end{array}$ & $2.7 \%$ \\
\hline $\begin{array}{c}\text { SUSTANCIAS } \\
\text { PÉCTICAS }\end{array}$ & $1.2 \%$ & PENTOSANOS & $10.5 \%$ \\
\hline $\begin{array}{c}\text { SUSTANCIAS } \\
\text { NITROGENADAS }\end{array}$ & $1.2 \%$ & LIGNINA & $11.3 \%$ \\
\hline MINERALES & $1.14 \%$ & CENIZAS & $0.7 \%$ \\
\hline OTRAS SUSTANCIAS & $1.32 \%$ & & \\
\hline
\end{tabular}

Cuadro comparativo de composición entre Fibra de Algodón y de Cabuya Fuente:(Hollen, 2004)

Para el análisis de procesos anteriores de suavizado de fibra de cabuya se realizaron los ensayos de laboratorio basándose en las siguientes fórmulas, siempre respetando la relación de baño de 1:50, las condiciones de temperatura $50^{\circ} \mathrm{C}$ durante todo el proceso y el tiempo recomendado de 40 minutos para la elaboración de las pruebas 


\begin{tabular}{|c|c|}
\hline PRODUCTO & CANTIDAD \\
\hline Suavizante Catiónico & $0.2 \mathrm{~g} / 1$ \\
\hline Acido Fórmico & $0.2 \mathrm{~g} / 1$ \\
\hline
\end{tabular}

\begin{tabular}{|c|c|}
\hline PRODUCTO & CANTIDAD \\
\hline Suavizante Catiónico & $0.5 \mathrm{~g} / 1$ \\
\hline Acido Fórmico & $0.2 \mathrm{~g} / 1$ \\
\hline
\end{tabular}

\begin{tabular}{|c|c|}
\hline PRODUCTO & CANTIDAD \\
\hline Suavizante Catiónico & $1 \mathrm{~g} / 1$ \\
\hline Acido Fórmico & $0.2 \mathrm{~g} / 1$ \\
\hline
\end{tabular}

\begin{tabular}{|c|c|}
\hline PRODUCTO & CANTIDAD \\
\hline Suavizante Catiónico & $1.5 \mathrm{~g} / 1$ \\
\hline Acido Fórmico & $0.2 \mathrm{~g} / 1$ \\
\hline
\end{tabular}

\begin{tabular}{|c|c|}
\hline PRODUCTO & CANTIDAD \\
\hline Suavizante Catiónico & $2.0 \mathrm{~g} / 1$ \\
\hline Acido Fórmico & $0.2 \mathrm{~g} / 1$ \\
\hline
\end{tabular}

\begin{tabular}{|c|c|}
\hline PRODUCTO & CANTIDAD \\
\hline Suavizante Catiónico & $2.5 \mathrm{~g} / 1$ \\
\hline Acido Fórmico & $0.2 \mathrm{~g} / 1$ \\
\hline
\end{tabular}

\begin{tabular}{|c|c|}
\hline PRODUCTO & CANTIDAD \\
\hline Suavizante Catiónico & $3.0 \mathrm{~g} / 1$ \\
\hline Acido Fórmico & $0.2 \mathrm{~g} / 1$ \\
\hline
\end{tabular}

\begin{tabular}{|c|c|}
\hline PRODUCTO & CANTIDAD \\
\hline Suavizante Aniónico & $0.2 \mathrm{~g} / 1$ \\
\hline Acido Fórmico & $0.2 \mathrm{~g} / 1$ \\
\hline
\end{tabular}

\begin{tabular}{|c|c|}
\hline PRODUCTO & CANTIDAD \\
\hline Suavizante Aniónico & $0.5 \mathrm{~g} / 1$ \\
\hline Acido Fórmico & $0.2 \mathrm{~g} / 1$ \\
\hline
\end{tabular}

\begin{tabular}{|c|c|}
\hline PRODUCTO & CANTIDAD \\
\hline Suavizante Aniónico & $1 \mathrm{~g} / 1$ \\
\hline Acido Fórmico & $0.2 \mathrm{~g} / 1$ \\
\hline
\end{tabular}

\begin{tabular}{|c|c|}
\hline PRODUCTO & CANTIDAD \\
\hline Suavizante Aniónico & $1.5 \mathrm{~g} / 1$ \\
\hline Acido Fórmico & $0.2 \mathrm{~g} / 1$ \\
\hline
\end{tabular}

\begin{tabular}{|c|c|}
\hline PRODUCTO & CANTIDAD \\
\hline Suavizante Aniónico & $2.0 \mathrm{~g} / 1$ \\
\hline Acido Fórmico & $0.2 \mathrm{~g} / 1$ \\
\hline
\end{tabular}

\begin{tabular}{|c|c|}
\hline PRODUCTO & CANTIDAD \\
\hline Suavizante Aniónico & $2.5 \mathrm{~g} / 1$ \\
\hline Acido Fórmico & $0.2 \mathrm{~g} / 1$ \\
\hline
\end{tabular}

\begin{tabular}{|c|c|}
\hline PRODUCTO & CANTIDAD \\
\hline Suavizante Aniónico & $3.0 \mathrm{~g} / 1$ \\
\hline Acido Fórmico & $0.2 \mathrm{~g} / 1$ \\
\hline
\end{tabular}

Luego de realizar las experimentaciones con los proceso tradicionales de suavizado de la fibra de la cabuya se determina que en todas las pruebas las solideces de las fibras no sufren ninguna variación o diferencia, es así que se puede considerar lo siguiente

\begin{tabular}{|c|c|}
\hline CARACTERISTICA & PARÁMETRO \\
\hline Densidad & $1,3 \mathrm{~g} / \mathrm{cm}^{3}$ \\
\hline Resistencia a la tracción & $305,15 \mathrm{MPa}$ \\
\hline Elasticidad & $7,5 \mathrm{GPa}$ \\
\hline Elongación a la fractura & $4,96 \%$ \\
\hline Absorción a la humedad & No tiene \\
\hline Higroscopicidad & Muy baja \\
\hline Resilencia & Deficiente \\
\hline
\end{tabular}

\section{Conclusiones Y Recomendaciones}


Al realizar las pruebas con los suavizantes tradicionales catiónicos y anicónicos la fibra de la cabuya se suaviza pero mantiene su aspecto áspero al contacto con la piel. En el caso del suavizante catiónico por ser un ácido graso en altas concentraciones da la característica de un tacto aceitoso a la fibra más no un tacto suave.

En el aspecto de suavidad se considera que la mejor muestra obtenida con el suavizante ácido graso es la de $2 \mathrm{~g} / \mathrm{l}$. En el caso del suavizante Anicónico en altas concentraciones mejora el tacto de la fibra de cabuya pero no le da cuerpo a la fibra

En el aspecto de suavidad se considera que la mejor muestra obtenida con el suavizante Anicónico es la de $3 \mathrm{~g} / \mathrm{l}$. Se debe procurar mantener la temperatura estable durante todo el proceso para poder conseguir un resultado confiable en las pruebas.

Cabe indicar que debido a los resultados obtenidos se propone el desarrollo de nuevas pruebas de suavizado, con productos que hasta el momento no han sido tomados en cuenta como las enzimas Celulasas de carácter ácido y neutro así como también con emulsiones y micro emulsiones de silicona

\section{Bibliografía}

Aguilar, Ramírez, \& Malnagon. (2007). Extracción de fibras no leñosas. Revista Iberoamericana de Políeros, 89-98.

Ardanuy, M. (2011). Fibras Procedentes de Recursos Renovables: una oportunidad para innovar y mejorar la competitividad. Revista de Química e Industria Textil, 24 - 29.

Avella, J. (2014). Aprovechamiento Residuos Biomasa De Producción De Piña. Ticson, 65 - 74.

Bastidas, \& Orozco. (2013). Materia Prima e industrialización. Arte Ancestral, 17-20.

Brown, E. (2010). Moda Ecológica o Ecomoda. Ocio, 36 - 40.

Castañeda, S. (2015). Moda Sostenible. ECODES, 57-61.

Cegarra, J. (1979). Introducción al Blanqueo de Materias Textiles. Barcelona España: Editorial Labor.

Centro de Estudios para el Medio Ambiente y el Desarrollo. (2007). El cultivo de Plantas Artesanales. Panamá: editorial Cemad.

Ekerlin, E. (1997). Ciencia Ambiental y Desarrollo Sostenible. México DF: International Thomson Editores.

Fletcher, K. (2012). Gestionar la sostenibilidad en la Moda. México: Blume.

Gobierno Nacional de la República del Ecuador. (2013). Plan Nacional del Buen Vivir 2013 - 2017. Quito Ecuador: Secretaria Nacional de Planificación y Desarrollo. 
González, J. (2013). La sostenibilidad ecológica en el desarrollo de productos textiles. Realidad y Reflexión, 65 - 97.

Hollen, N. (2004). Introducción a los Textiles. México DF: Mc Graw Hill.

Jurado, s., \& Sarzosa, X. (2009). Estudio de la Cadena Agro Industrial de la Cabuya en la Producción de miel y licor de cabuya. Quito Ecuador: Editorial de la Escuela Politécnica Nacional.

LCI Bogotá. (2011). Textiles Ecológicos: Reduciendo el impacto Ambiental de la Industria Textil. LCI, 35-40.

Manfred, P. (2015). Terminación Textil Química Aplicación y Tecnología. Sao Paulo, Brasil: CHT BEZEMA.

Ministerio de Agricultura y Ganadería. (2008). La cabuya: Cultivo e Industrialización. Texas Estados Unidos: Editorial del Ministerio de Agricultura y Ganadería.

Morales, N. (2002). Guía Textil en el Acabado. Ibarra Ecuador: Editorial Universitario Universidad Técnica del Norte.

Orejuela, E. (1996). Teñir la cabuya proceso facil. Bucaramanga Colombia: Ministerio de Agricultura y desarrollo rural, Centro de investigaciones en celulosa palpa y papel, Universidad Industrial de Santander.

Planeta Moda. (2016). Fibras Textiles También En Ecológico. Vida Sana, 28-35.

Pro humana. (2010). Textiles ecológicos para confecciones sustentables. Pro Humana, 7-10.

Ramírez, J. (2014). Hilatura fina. Revista de la Cámara artesanal de Quito, 6-7.

Red Textil Argentina. (25 de 03 de 2012). Red Textil Argentina. Obtenido de Red Textil Argentina: www.redtextilargentina.com.ar/

Rivas, M. (2015). El cuero a base de fibras de piña que revolucionará el mundo textil. VICE, 50-57.

Salcedo, E. (2014). Moda Ética para un futuro sostenible. Madrid, España: Editorial Gustavo Gill.

Sánchez, A. (1990). Cultivo de Fibras, manual para educación agropecuaria. México D.F., México: Editorial Trillas.

Tapia, F. (1995). Medio Ambiente ¿Alerta Verde? Madrid España: Editorial Acento.

Yépez, 1., \& Esparta, M. (2009). Perfil de las demás cuerdas y cordajes del género agave: fibras de Cabuya, Sisal, etc. Quito: Centro de información e inteligencia Comercial, Pontificia Universidad Católica del Ecuador. 